\title{
The World in an Equation: A Reappraisal of the Lemaître's Primeval Cosmic Rays
}

\author{
Russell Bagdoo \\ Saint-Bruno-de-Montarville, Quebec, Canada \\ Email: rbagdoo@gmail.com,rbagdoo@yahoo.ca
}

How to cite this paper: Bagdoo, R. (2019) The World in an Equation: A Reappraisal of the Lemaître's Primeval Cosmic Rays. Journal of Modern Physics, 10, 922-952. https://doi.org/10.4236/jmp.2019.108061

Received: May 29, 2019

Accepted: July 6, 2019

Published: July 9, 2019

Copyright $\odot 2019$ by author(s) and Scientific Research Publishing Inc. This work is licensed under the Creative Commons Attribution International License (CC BY 4.0).

http://creativecommons.org/licenses/by/4.0/ (c) (i) Open Access

\begin{abstract}
Based on radioactive phenomena (weak force), Georges Lemaître conceives, as soon as 1927, the primeval universe as a "unique super-dense quantum", whose disintegration gave birth to all the current components of the universe [1] [2]. Using quantum mechanics, he proposes to explain the origins of the world from the point of view of quantum theory. He believes to find in the cosmic rays the manifestation of the initial fragmentation. However, regardless of the adopted cosmology, the hypothesis of the primeval atom (cold big bang) had no equation to support it and was not retained. Like all other cosmologists, he fell back on the Friedmann-Einstein equation with a repulsive cosmological constant which, according to supernova observations at the end of the millennium, propels expansion towards infinity. We juxtapose our equation of "quantum cosmology" to this equation of relativistic cosmology. We have already proposed this equation in an earlier paper [3], which has its source in quantum mechanics and fits Lemaitre's hypothesis of the "primeval atom". It's an equation in which the concept of matter-space-time is mathematically connected; gravitation and electromagnetism are also bound by space-time. A mechanism is described showing how velocity, time, distance, matter and energy, are correlated. We are led to ascertain that gravity and electricity are two distinct manifestations of a single underlying process: electrogravitation. For the first time, the cosmological time, considered as a real physical object, is integrated into a "cosmological equation" which makes coherent what we know regarding the time (its origin, its flow...), the matter and the space. Moreover, the equation indicates a constantly decelerated expansion. The concentration of the material medium and the importance of the decreasing energy of the vacuum contribute to the progressive increase of the positive pressure which becomes responsible for the increasing deceleration of the expansion. Does this mean that our equation leads us inevitably to the hypothesis of the primeval atom for the whole cosmos? Certainly not, since our model includes both the hot Gamow model and the cold Lemaitre
\end{abstract}


model. The term "dynamic evolution" (used in the beginning by specialists for big bang models) is appropriate for our model since there is both an explosive origin and, throughout the expansion, a disintegration of a hyper-dense matter. The discovery of cosmic microwave background radiation has confirmed the hot big bang model that Gamow and his team have achieved. The predicted light prevailed over the primitive cosmic rays (particles) suggested by Lemaitre. Nevertheless, we think that Lemaitre was also right. The so-called big bang theory (singular cataclysmic explosion), in addition to not meeting basic criteria of science, is contradicted by several observations that are ignored. For example, the work of Armenian astronomers has convinced us that the origin of cosmic particles results not only from supernova explosions, but also from the partition of radio galaxies, not only from the death of the world, but also from their birth.

\section{Keywords}

Theory of Relation, Irreversible Cosmological Time, $\pi$, Deceleration, New Variable, Quantum Cosmology, Primeval Atom, Cosmic Rays

\section{Introduction}

The standard model of the big bang theory, in its main features, is widely distributed in the general public, at the risk of doing that the history of the universe is from now on an acquired knowledge. To describe our universe, the cosmological model relies on fundamental laws supposed to describe all the phenomena of nature, which it extrapolates to cosmic scales. But, in order for this model to be in agreement with all the astronomical observations (the acceleration of the expansion of the universe highlighted in 1998), it was necessary to introduce a dark energy of which no physical theory explains the origin.

If general relativity describes gravitation at cosmological scales, then the expansion of the universe can be accelerated only under one condition: the matter that dictates the dynamics of the universe today must be such that the sum its energy density and of three times its pressure is negative. For this, a repulsive black energy has been inserted in the Einsteinian equations. This new material component incorporated in the model in the form of a cosmological constant would be only a manifestation of the quantum fluctuations of the vacuum. The calculations lead to a value between $10^{60}$ and $10^{120}$ times greater than that deduced from cosmological observations. Which is a real disaster!

But this does not end here. When, in 1998, cosmologists announced that, based on a study of 21 Type-1A supernovae, the very fabric of space was expanding, they concluded that they were a proof of a positive cosmological constant and a hitherto unsuspected dark energy that accelerated the expansion of space [4]. In our view, it was a botched and biased study to preserve the construction of the standard cosmological model. In a previous article about the Pioneer effect, we said the deceleration of the probe in distant spaces where the 
wavelength of space-time grows is in itself an experimental proof of a world in deceleration [5]. In another article on the positive cosmological constant starting from type la supernovae observations, we showed that its official accreditation in 1998 was premature and misinterpreted [6].

Subsequently, by adding type 1a supernovae, astronomical teams confirmed these data, others revealed systematic uncertainties, and no clear evidence was found for a possible evolution of the slope (beta) of the color-luminosity relation with the redshift [7], direct evidence of dark energy rather weak [8], serious doubts about the acceleration of the universe [9], statistical analysis of supernovae data-set that leaves much to be desired [10], results consistent with a cosmological constant that give only weak constraints on a w that varies with redshift [11], etc. Despite serious reservations, three astronomers received the Nobel Prize in Physics in 2011 for their discovery that the universe was growing at an accelerated rate.

In 2016, an international team of physicists, approaching the problem with a fresh look, questioned the acceleration of the expansion of the universe [12]. As Subir Sarkar, a researcher at Oxford University, reports: "We analysed the latest catalogue of 740 Type Ia supernovae-over 10 times bigger than the original samples on which the discovery claim was based-and found that the evidence for accelerated expansion is, at most, what physicists call ' 3 sigma'. This criterion is far short of the ' 5 sigma' standard required to claim a discovery of fundamental significance." Instead of finding evidence to support the accelerated expansion of the universe, Sarkar and his team say it looks like the universe is expanding at a constant rate [13].

If expansion is constant, drawing new physics with dark energy is not necessary. Does this also mean that the fundamental principles of the standard cosmological model do not have to be questioned? They must be called into question because, despite the consolidations for the big bang theory and the setting of the fundamental parameter values for thirty years, it is a model "weaken" by the theory of inflation in effect. Recall that the satellite XMM-Newton of Agency's European Space X-ray observatory (ESA) [14], has returned data about the nature of the universe indicating that the universe must be a high-density environment, in clear contradiction to the "concordance model" (according to which the universe is today composed of about $73 \%$ of dark energy) linked to the theory of inflation (whose origin is unknown). In a survey of distant clusters of galaxies, the results of XMM-Newton revealed that today's clusters of galaxies are superior to those present in the universe around seven thousand million years ago. Such a measure also goes toward a decelerated expansion [15].

The acceleration of the universe and the repulsive dark energy are the two components of the inflation theory, which was supposed to be the miracle cure for the pathology of the causality of the standard model. It seems that the reme$\mathrm{dy}$ is worse than the disease. There is no convincing theoretical explanation for the existence of dark energy, its nature or its magnitude. The so-called accelera- 
tion of the universe only further demonstrates that the theories of fundamental particles and gravity are incorrect or incomplete. Most experts believe that it will take nothing less than a revolution in our understanding of fundamental physics to achieve a complete understanding of cosmic expansion. For these reasons, we propose our quantum cosmological model.

It contains an equation, developed in an earlier work [3], which is doubly hybrid: quantum and relativistic by its construction, revealing and embarrassing because of its consequences. If it elegantly gives a natural place to cosmological time, it reveals objects that are hated: negative energies. The purpose of this paper, besides the exposure of this equation, is to present the hypothesis of the primitive atom of Georges Lemaitre at the origin of the concepts of expanding universe and big bang. Lemaitre had anticipated the fundamental role played by quantum mechanics, vacuum energy and the existence of a fossil radiation. In the absence of an equation to support his vision, he adopted the Einstein-Friedmann equation, which led to the current acceleration of expansion. We think that our equation is the one that Lemaitre was missing from the start.

This paper is divided into four parts, intended to be complementary. In 2.1 and 2.3 the equation of the theory of Relation presents the universe as an expanding super-atom. In 2.2 we emphasize the importance of pi in the equation. In 2.4 expansion... and yet it decelerates! In 3.1 we discuss the hypothesis of the primeval atom of Georges Lemaitre. In 3.2 some features of the history of the standard big bang. In 3.3 the hypothesis of the primeval atom of Lemaitre is confronted with that of the primitive atom of Gamow. In 3.4 we discuss about Lemaître's primeval cosmic rays. In 3.5 we discuss to find out if Lemaitre's cold model is as true as Gamow's hot model. In 4.1 we show how the cosmological time in our equation links the physics of the infinitely large to that of the infinitely small. In 4.2 we browse Planck's units through our equation. In 4.3 we present $M_{V P}^{2}$, the new essential parameter. In 5 we list the advantages of this quantum cosmological model and its equation which gives the same results as those obtained with the classical models which refer to the Einstein-Friedmann equation. Nature of our universe: everything happens as if there were two universes in one; the expansion would have been preceded by a period of contraction and it would not have occurred at the same time for all matter. We emphasize that the standard big bang theory refuses to take into account the existence of negative energy particles, thus denying half of our universe. It is also contradicted by several observations left aside, for example the works of Armenian astronomers whose observations have confirmed the hypothesis of the formation of stars according to which evolution was made of hyper-dense bodies to less dense bodies. These astronomers have furthermore validated that the birthplaces of the new galaxies were the centers of the old galaxies, as well as the theory of the division of galaxies. In 6 we conclude that this equation is the one which gets closer most to the equation which missed to Lemaitre to defend his "hypothesis of the primeval atom" and his prediction of fossil cosmic rays. 


\section{Equation of the Theory of Relation}

\subsection{The Equation of the Theory of Relation}

Historically, Newton's discovery of the law of gravitation can be appreciated as the first "unification", combining the laws of heaven and earth. The next great leap took place in the mid-1860's with Maxwell's theory of electromagnetism uniting electricity and magnetism. In 1905, Einstein created the special theory of relativity connecting space and time and associating the concepts of matter and energy. In 1915, he proposed general relativity, which explained gravitation as the marriage of space-time and matter-energy. In the 1960's, the works of S. Weinberg, A. Salam and S. Glashow led to the unification of the electromagnetic interaction and the weak nuclear interaction. The next step, namely the unification of the electroweak and strong interactions, drove to the electronuclear theory (GUT) whose predictions were the object of no conclusive result. As for the ultimate synthesis-the unification of gravitation and GUT-, it has defied all attempts [16].

More than seventy years ago, Paul Dirac suggested that more than a coincidence was at work between the age of the universe in atomic time units and the ratio of the electric force between an electron and a proton to the gravitational force between the two $\left[k e^{2} /\left(G M_{p} M_{e^{-}}\right)=10^{40}\right]$ [17] [18]. The most fundamental unit of time would be one associated with atomic processes, because it would depend only on basic natural constants, such as the electric charge (e), the mass of the electron $\left(M_{e_{-}}\right)$, or the speed of light (c). This time unit, which appears throughout physics as the basic time scale for atomic and nuclear processes, is roughly the time required for light to travel the electron radius: $10^{-15} \mathrm{~m} / 10^{8} \mathrm{~s}=10^{-23} \mathrm{~s}$. Thus the evaluated age of the universe $\left(10^{17} \mathrm{~s}\right)$ in atomic time units is $10^{17} \mathrm{~s} / 10^{-23} \mathrm{~s}=10^{40}$. Dirac postulated that the near equality of these two numbers was a manifestation of some as yet the unknown deeper law of nature that required them to be nearly equal for all time.

The problem is that the age of the universe is increasing. If the quantity between the two $10^{40}$ is to be maintained, then one of the other numbers must change with time. For many physicists, the gravitational constant $(G)$ seems the only plausible candidate which can vary in spite of general relativity, which states that $G$ is a physical constant whose numerical value is fixed.

Let us compare the electrostatic and the gravitational forces between two protons in the same nucleus, with a distance of 0.2 nanometers [19]. We will use the MKS system which has the advantage of incorporating the constants of the permittivity of free space and of permeability of free space. The value of the Coulomb constant $k$ is $1 / 4 \pi \varepsilon_{o}=8.9875 \times 10^{9} \mathrm{~N} \cdot \mathrm{m}^{2} / \mathrm{coul}^{2}$. The value of the constant $\varepsilon_{0}$, called permittivity of free space, is $8.8542 \times 10^{-12} \mathrm{coul}^{2} / \mathrm{N} \cdot \mathrm{m}^{2}$. According to Coulomb's law, the electrostatic repulsive force is

$$
F_{e}=q^{2} /\left(4 \pi \varepsilon_{o} R^{2}\right)=5.775 \times 10^{9} \mathrm{~N} ; e^{2} /\left[4 \pi\left(8.8541878 \times 10^{-12}\right)\left(0.2 \times 10^{-9}\right)^{2}\right] .
$$

The attractive Newtonian force is $G M_{o p}^{2} / R^{2}=4.666 \times 10^{-45} \mathrm{~N}$. The ratio is 
$F_{e} / F_{g}=k e^{2} / G M_{p}^{2}=1.23 \times 10^{36}$.

Let us pursue Dirac's suggestion on the time, and replace the ratio by a universal time factor with the constants $G$ and $c . F_{e} / F_{g}=t_{o} c / G ; F_{e}=F_{g} t_{o} c / G$. And suppose we relativize the masses of the protons, in accordance with special relativity, as if they were moved with a speed of $200,000 \mathrm{~km} / \mathrm{s}$, we would obtain

$$
\begin{aligned}
& k e^{2} /\left[R_{o}\left(1-v^{2} / c^{2}\right)^{\frac{1}{2}}\right]^{2} \\
& =G\left[M_{o p} /\left(1-v^{2} / c^{2}\right)^{\frac{1}{2}}\right]^{2} /\left[R_{o}\left(1-v^{2} / c^{2}\right)^{\frac{1}{2}}\right]^{2}\left[t_{o} c / G\right],
\end{aligned}
$$

thus we would have

$$
k e^{2}=M_{V P}^{2} t_{o} c .
$$

[ $M_{o p}$ is rest-mass; $M_{o p}\left(1 /\left(1-v^{2} / c^{2}\right)^{1 / 2}\right)$ gives $M_{v p}$, i.e., rest-mass + kinetic energy $(T) ; \quad v=200000 \mathrm{~km} / \mathrm{s}=2 / 3 c$ ].

Particles come in pairs, each with a counterpart antiparticle

$$
\begin{gathered}
\pm k e^{2}= \pm\left[M_{\text {op }} /\left(1-v^{2} / c^{2}\right)^{1 / 2}\right]^{2} t_{o} c \\
2.3 \times 10^{-28} \mathrm{~kg} \cdot \mathrm{m}^{3} \cdot \mathrm{s}^{-2}=\left(2.2439 \times 10^{-27} \mathrm{~kg}\right)^{2}\left(1.528 \times 10^{17} \mathrm{~s}\right)\left(3 \times 10^{8}\right) .
\end{gathered}
$$

We ascertain that the link between the charge squared and the relativized proton's mass squared confers a universal time of $1.5283 \times 10^{17} \mathrm{~s}$ multiplied by $c$. That time gives 4.84 billion years $\left[\left(1.5283 \times 10^{17}\right) /(365.24 \times 24 \times 60 \times 60)\right]$.

\subsection{The Importance of $\mathrm{pi}$}

We have already talked about pi in a previous article [3]. We considered that pi made an essential difference between a linear time in accordance with a longitudinal wave and a circular time which refers to a transverse wave. A particle that travels 4.84 billion years in the metric of a straight-line space will travel the same Euclidean distance in $\mathbf{1 5 . 2 1}$ billion years using the metric of a space with constant curvature. We imagined that a wave rolled up around the radial line A-Z would travel it in the 5.21 billion years, which is linear time multiplied by $\pi$. It fits a transverse electromagnetic wave

$$
k e^{2}=M_{V P}^{2}(\pi) t_{o} .
$$

Of this expression, one must keep in mind that $\pi$ is used for winding the particle spirally around the radial length $t_{o} c$. It could be a transverse electromagnetic wave but it could also be a transverse gravitational wave. Mathematically, the equation should be

$$
(\pi) k e^{2}=M_{V P}^{2}(\pi) t_{o} c .
$$

This way of seeing predicts the existence of transverse and longitudinal electromagnetic waves, as well as transverse and longitudinal gravitational waves. The particles that may be associated with longitudinal electromagnetic waves 
and longitudinal gravitational waves could be the neutrino and the graviton. The longitudinal electromagnetic wave already exists. The gravitational wave, which has always been considered to be transverse, would have been captured in 2015. This does not exclude the existence of a longitudinal gravitational wave.

This article describes pi in another way. The reason is that it makes it possible to obtain other forecasts. We find that the case of a transverse wave in relation to a longitudinal wave is very similar to that of the light propagating on the circumference of a circle, or on the surface of a sphere, whose pi makes it possible to determine its radius. Although the two descriptions seem to be very different from each other, there is a mathematical equivalence between them $\left[\alpha=\pi R=\pi t_{o} c\right.$; $\alpha$ can represent a semicircle, a transverse path, a transverse wave; $R$ can represent a radius, a radial path, a longitudinal wave].

When Einstein's geometric theory of gravitation is applied to the entire universe, space is curved on a global scale. This curvature results in geodesics and the light ray (or photon) is the ideal tracer of geodesics. The curvature of the universe on a cosmic scale is manifested by the fact that the real mutual distance between two galaxies located at the antipodes of each other will be equal to the product of $\pi$ by the radius $R: \quad \alpha=\pi R$. What fixes the scale of the curvature is the inner radius of the universe because we consider the universe as a sphere having a volume and not only a surface. This "geometric" way of representing $\pi$ makes it possible to obtain a central point, an origin, a privileged direction. While the Einsteinians can assert that a point on the surface of the universe is everywhere a center of the universe, we can say that any point on the surface of the universe has the same center of the universe. The center of the sphere becomes a unique, privileged direction.

With the theory of Relation, the radius $R$ of the universe gives on the center of the universe, towards the original point of our universe. This model considers our universe to be spherical, expanding, with a surface that is curved, finite, and boundless [20]. It gives its approximate age, its past and future history starting from microscopic units based on atomic data. The model of the theory of Relation follows the cosmological principle of homogeneity and isotropy and the law of raisin cake. In a cake of raisins that swells while cooking, the raisins move away from each other not because they move in the dough but because it increases in volume and at the same time grows the mutual distance between any two raisins. It is the space between the raisins which increases, it is not them who move in the dough. The term $t_{o} c$ in the equation represents the dough of the cake being inflated, it is the radius of the universe which grows and remains unobservable, it is a cosmological dark energy whose wavelength follows the size of the space. The wavelength of this energy-radiation propagating through the space-time it creates varies as the size of the universe and is expressed by the cosmological redshift. What is observable are the galaxies, that is to say the raisins. It is not the galaxies that are in motion, it is the space between the galaxies 
that is expanding [21].

It can be said that with expansion, galaxies are at the edge of the universe. We are part of a galaxy and we measure the universe through light from other galaxies. If we leave the edge of the universe, what we call its surface and go straight away, as a light ray would do, along a geodesic, we would eventually reach the farthest point, which we call the anti-center.

This point is situated at a certain distance $\alpha$, and the elementary geometry teaches us that the distance a from one pole to the other is equal to the product $\pi$ by the radius $R$

$$
\alpha=\pi R .
$$

In the opposite direction, if we do not know what the radius $R$ is, it suffices to divide the distance $\alpha$ between a pole and the opposite pole, that is to say the point which is the furthest away, by the number $\pi$. This definition is applicable to our universe. This internal radius will be

$$
R=\alpha / \pi \text {. }
$$

We consider the universe as a finite object without limits. The circumference of a circle and the surface of a sphere are examples of one- and two-dimensional spaces that are finite but have no beginning or end. One can imagine a four-dimensional mathematical sphere, a hypersphere, of which the three-dimensional universe constitutes the surface, or rather the hypersurface. Just as the circle and the sphere are equidistant from a fixed point of the space called the center, so the hypersphere is made of a three-dimensional distribution of points, all situated at the same distance from the center [22]. The three-dimensional volume of the hypersphere is: $2 \pi^{2} R^{3}$ [Volume $=$ area $\left(\pi R^{2}\right) \times$ circumference $(2 \pi R)=$ $\left.2 \pi^{2} R^{3}\right]$.

By moving on the surface of a sphere (along a meridian, for example) the light would eventually return to its starting point, having traveled the distance $2 \pi R$. The distance a from one pole to the other, a half circumference, is equal to the product $\pi$ by the radius $R$ (which is $t_{o} c$ in our equation). The radius $R$ is $\alpha / \pi=t_{o} c$. If one admits an explosive origin, the ray starts from the center of the sphere in all directions, looping the 360 degrees of the surface. There is a simultaneity of time between the ray which reaches the point which forms the surface and that surface which is formed, since the ray comes everywhere from the same origin. In the expression $\alpha=\pi R=\pi t_{o} c$, the time is the same to obtain $R$ and $\alpha$, but the distances are different, which suggests a curved electromagnetic distance for $\alpha$ and a longitudinal radial distance for $R$.

We measure the universe thanks to the light that comes from the stars. This light follows a geodesic to reach us. The estimated age of the universe is about 15 billion light-years and its radius is $10^{26}$ meters. This geodetic ray $(\alpha)$ of 15 billion light-years has an internal radius $(\alpha / \pi)$ with a time of 4.5 billion light-years.

For the Einsteinians, the universe-sphere is a false image that seeks to impose 
itself on our mind and the radius of the universe in relation to the big bang is not the radius of a ball. They seem to know only the universal space of the surface that surrounds the solid globe, and which constitutes the three-dimensional universe on which we could go where we want and visit all of its galaxies. Everywhere the space would be the same, we would never meet any edge opening on an outside. This universe is finite but without borders. It has no exterior, and even less interior. It is their truth, but their very truth that carries its nonsense by wanting to consider the universe only for the surface that surrounds it. The universe must be considered for itself in its volume. We can measure its size using its internal radius, understood as the distance from a point of the surface to its anti-point divided by $\pi$.

\subsection{Return to the Equation of the Theory of Relation}

Equation (4) establishes a clear mathematical link between electromagnetism and gravitation. It takes into account $\pi$, as if $t_{0} c$ was a transverse space-time wave. Equation (3), in the form

$$
\pm k e^{2}= \pm M_{V P}^{2} t_{o} C
$$

does not take account of $\pi$, as if $t_{o} c$ was longitudinal. We will not take into consideration $\pi$ for relativistic expressions of the rest of the paper.

In the right-hand side, matter $\left(M_{v p}^{2}\right)$, space $\left(t_{o} c\right)$, and time are linked into one whole. The radius of the universe is represented by " $t_{0} c$ ". We can see in relation (1) that the term $G\left[M_{o p} /\left(1-v^{2} / c^{2}\right)^{1 / 2}\right]^{2} /\left[R_{o}\left(1-v^{2} / c^{2}\right)^{1 / 2}\right]^{2}$ links the gravitational Newtonian force and special relativity. We obtain a relativized Newtonian gravitation [23], which means, on the one hand, that gravitation is a reality everywhere and, on the other hand, that special relativity is neither only a mathematical tool nor a simple Galilean reference without gravity. Both are linear theories applied to a linear three-dimensional Euclidean geometry with flat space-time. Let's add that electromagnetism is also a linear theory.

According to the theory of Relation, gravity is not a distinct force, but an aspect of electromagnetism. The two forces are connected by space-time in four dimensions. In fact, gravity is electromagnetic dissolution in space-time. Basically, electric and gravitational forces are part of a common super-force: electrogravitation. As magnetism and electricity are both sides of electromagnetism [24]. In the physics of subatomic particles, electrogravitation takes the appearance of the electrostatic force, and the gravitational force, $10^{36}$ weaker, plays no apparent role in it. When the pair of particles with its two positive electric charges repel and move away at a speed close to light, creating the "space-time" between them, electromagnetism decreases with distance and becomes the vacuum energy. Its lost energy has turned into attractive energy, that of gravitation that grows with space-time. On a large scale, electrogravity has become gravity. On a large scale, electrogravitation becomes gravity. We are led to think that attractive gravitational forces are electromagnetic forces with attractive charges 
acting in space-time rather than in the subatomic world. The expansive driving force, caused by the initial explosion, would be the electromagnetic forces of the repulsive forces operating in the universe.

According to Newton's theory of gravitation, the force $G M m / r^{2}$ instantaneously transmits energy or a signal. Newton was unhappy with an instantaneous phenomenon, or a "remote action," associated with gravity. Poincaré (1904), Minkowski (1908), and de Sitter (1911) agreed that gravity must propagate at the speed of light. Indeed, according to special relativity nothing moves faster than light, not even gravity. None of the several theories of gravity-even Einstein's, which were compatible with special relativity in that the speed of propagation of gravity is the speed of light, was satisfactory. The combination of the Lorentz transformation and $t_{o} c$, ensures that the speed of light or gravity does not go faster than the speed of the universal constant $\mathrm{c}$.

However, the gravitational constant $G$ disappears in the equation, which implies that the classical gravitational mass of matter at the beginning is in the form of a minimum potential while the energy is at its maximum [25]. This goes against Paul Dirac who, in papers published in Nature in 1937 and in the Proceedings of the Royal Society in 1938, described a cosmology with a changing gravitational constant. He postulated that $G$ varies like the inverse age of the universe, so as the universe expanded from the big bang, the gravitational constant, or force, became weaker and weaker as time passed until today, when we experience the present very weak force of gravity [26]. With the theory of Relation, Newton's gravitational constant $G$ does not vary, just as in general relativity. On the other hand, potential energy-mass increases with cosmic time [27].

The equation of the theory of Relation is in phase with the Englert-Brout-Higgs proposition allowing to reconcile the equations of the standard model with the empirical data. It consisted in postulating the existence of a quantum field filling the whole space, with which the elementary particles, effectively without mass, interact more or less strongly, which has the effect of slowing down their movements in the same way as if they had a mass. Everything happens as if the elementary particles were massless objects at the beginning of the expansion, moving on an electromagnetic field (or an electromagnetic space-time wave-amalgamated with the vacuum energy, at the cosmological constant [6] and dark energy) that loses energy over time. This lost energy is recovered by the particles that move more and more with friction, so at a speed less than that of light and their mass is non-zero. In the equation, the mass then appears as a measure of the velocity decrease of the matter ( $v$ of $v^{2} / c^{2}$ which decreases throughout the expansion), the inertia, the resistance to movement, mass.

The equation is remarkably that of expansion. Imagine that the proton masses of our equation that we have relativized above, as if they were moving at a speed of $200,000 \mathrm{~km} / \mathrm{s}$, are galaxies moving away at $2 / 3 c$, we then obtain "a cosmological equation" which establishes the age and distance of the universe in relation to the speed of recession of galaxies. The farther the galaxy is from the earth, the 
greater the velocity of recession, and the younger the age of universe. Velocity, age and distance are correlated. A speeding away of the galaxies at $2 / 3 c$, taking account of $\pi$, is tantamount to 15 billion years. These two numbers are roughly fitting with the actual valuations of science.

If we admit that the universe is a kind of expanding super-atom:

$$
k e^{2} \rightarrow M_{V P}^{2} t_{0} c,
$$

giving the age of the universe, we have an arrow toward the future being the same as at least 3 arrows of time that do distinguish the past from the future: thermodynamic (disorder increases); cosmological (universe expands rather than contracts); psychological (we remember the past, not the future) [28].

\subsection{Two-Edged Reasoning}

The distant supernovae serve as luminous standards for surveying the universe on a large scale. The gigantic explosion of a voracious white dwarf makes visible an intense light that persists for several days. Their curves of light are similar. It has been deduced that any difference between two curves of light can only come from distance: the further away the supernova is, the weaker the received light. The results obtained showed that the light of distant supernovae was $25 \%$ fainter than expected in case of deceleration. The majority of astrophysicists have therefore concluded that the expansion of the universe has been accelerating for several billion years and that a repulsive dark energy plays the role of accelerator [29].

$A$ contrario, if we assume a deceleration of the expansion, this means that the transformations accelerate towards the past and that the intrinsic luminosity can no longer be theoretically always the same and that we cannot be satisfied with measure their apparent luminosity to deduce their distance. The physical conditions change by going as far as possible, the speed rating of chemical, atomic and nuclear reactions had to be faster. The explosions of these stars were to occur when they reached a critical mass different from the supernovae that are close. They release a different amount of energy, their radiations are modified. They emit less luminosity because the mechanisms are too fast or skip steps. It can be assumed that the peak of brightness may last less and the subsequent weakening. As for the apparent luminosity, one can conjecture its degradation by the galactic dust, in particular the iron needles produced by condensation of the iron rejected by preceding generations of supernovae [30].

Whatever the current scientific consensus, the fact remains that the same results obtained (pallor greater than expected) show that these supernovae are no more distant than those predicted by classical cosmological models. They show that the explosion of the universe, contrary to what has been imagined since 1998, has been in a deceleration phase since the Planck era [6]. Which is consistent with the equation of the theory of Relation.

What does it mean for the dark energy that must play a role of accelerator as if a sort of antigravity forced the universe to constantly increase the speed of its expansion? It exists but differently. It is nothing else but the gigantic kinetic 
energy of the universe when it started in a "cosmic fireball". It has become today the vacuum energy [31]. The logic of this cosmological adventure revolves around an essential property: the progressive de-excitation of the quantum field by the decelerated expansion of space [32]. There is a snowball process. The deceleration of space precipitates the rate of condensation of the matter energy, which reduces the influence of the vacuum energy, which dilutes and decreases, in return, the rate of expansion, which leads to a universe in which clusters of matter become very dense. In the context where dark energy and dark matter do exist, we believe that their proportion to constitute matter must be reassessed. We expect, by keeping the ordinary visible matter at about $4 \%$, that the dark matter would be about $45 \%$ and that the dark energy, which was diluted with the expansion of the universe, is about $50 \%$. (consistent with the hypothetical $2 / 3 \mathrm{c}$ velocity of our equation and with a constant rate of expansion [12]).

\section{The Hypothesis of the Primeval Atom of Georges Lemaitre}

\subsection{The Primeval Atom of Georges Lemaitre}

The term $M_{V P}^{2}$ dovetails with Georges Lemaitre's hypothesis that the universe comes from a kind of gigantic atomic nucleus containing all the nucleons of the universe, a nucleus whose decay would have initiated the expansion of the universe [33]. Lemaitre believes that the cosmic rays, which are endowed with energy of several billion electrons-volts, are the manifestation of initial fragmentation.

Although Lemaitre's idea of explaining the expansion of the universe as being due to an initial explosion is still relevant, his theory that the entire universe was originally contained in a single atom that has disintegrated is now relegated to oblivion. He had wished to give an experimental basis to his hypothesis of the primitive atom, and he believed find in the cosmic rays the relics of the primitive universe. He had deepened the question with collaborators such as Odon Godart (1913-1996) and Manuel Sandoval Vallarta, but the failure of their explanation will not favor the credibility of the model of the primeval atom [2].

Physicists are now leaning towards a sort of cloud of elementary particles (quarks and leptons) that have condensed gradually, which has released energy and given the universe its initial impetus. They recognise the existence of fossil radiation, a trace of the initial explosion, but which no longer comes, as Lemaitre thought, from a drag of particles propelled by the disintegration of the original atom but from electromagnetic radiation [34]. It was therefore concluded that Lemaitre was mistaken. In light of the developments that followed, one can question, without excluding it, his model of the primitive atom. But we believe he was not mistaken about cosmic rays: fossil cosmic rays from the beginning exist just as much as fossil light. But to claim it, it is necessary to revisit the history of standard big bang.

\subsection{Some Features of the History of the Standard Big Bang}

In 1929, Edwin Hubble discovered the law of recession of galaxies thanks to the 
telescope placed on Mount Wilson: they move away from each other at a speed that is greater as their distance is greater, proof that the universe is expanding and not static. Until then, Einstein believed in a static universe. In 1922, Friedmann, by dynamically interpreting Einstein's equations, had provided the first expanding universe model with positive curvature and density, a nonzero cosmological constant, and zero pressure. Regardless of Friedmann, Lemaitre in 1927 suggested, with calculations of general relativity in support, that the universe was expanding: he gave the first interpretation of the redshifts related to the expansion of the universe and predicted the linear relation distance-shift to red. Friedmann and Lemaitre had put forward this hypothesis before it was confirmed by the observations of redshift of galaxies and the Hubble law. Contrary to what Hubble himself imagined, it is not the galaxies that move, but the space itself that extends, taking with it the galaxies. Hubble experimentally establishes the linear relationship distance-redshift, but has not linked it to expansion. Einstein, Friedmann, Lemaitre and Hubble were the pioneers of relativistic cosmology. The latter is essentially based on the Friedmann-Einstein equation.

In 1931, Lemaitre distinguished himself by proposing a quantum origin of the universe. He proposed his initially singular universe model, the primitive atom, in which a phase of stagnation allows the formation of galaxies. He suggested that cosmic rays could come from radiation produced during decays during the first expansion period. This hypothesis, prefiguration of big bang models, left physicists very skeptical. Einstein and others blamed this hypothesis for having been inspired by the Christian dogma of creation. Lemaitre often expressed that the physical beginning of the world was quite different from the metaphysical notion of creation. The irony is that in the same year Einstein published an article in which he admits that the observations establish without any doubt that the universe is expanding. The whole scientific community ranked behind the models describing an expanding universe only in 1964, the year in which they received a start of confirmation thanks to the discovery of the cosmic microwave background [2] [34].

\subsection{The Hypothesis of the Primitive Atom of Lemaitre Confronted with That of the Primitive Atom of Gamow}

In 1945, Lemaitre assembled his cosmological work in "The hypothesis of the primeval atom" [35]. It is a cosmogonic hypothesis according to which the present world has resulted from the radioactive decay of an atom. He was led to formulate it by being guided by thermodynamic considerations that sought to interpret the law of energy degradation in the context of quantum theories. The discovery of radioactivity and the establishment of the corpuscular nature of cosmic rays have made plausible its hypothesis that assigned a radioactive origin to these rays as well as to all existing matter.

This hypothesis had to compete with that of George Gamow. The latter also tackled the fundamental problem of the origin of our universe. He wondered 
why our universe was in a state of such high compression and what triggered the expansion? His answer: The great compression that occurred at the beginning of the history of our universe resulted from a collapse which had taken place in an even older time, and that the current expansion is simply an "elastic" rebound that began as soon as the density corresponding to the maximum possible compression was reached. We can presume over the pre-compression era, but we can say that as soon as the density of the universe reached its maximum value, the direction of motion reversed (which is why the negative energy belongs to before the big compression and the positive energy to after) and expansion began, so that the very high densities probably existed for a very short time. The theory of Relation shares this cyclical vision [36].

In fact, it is especially with regard to the nuclear forces that the two hypotheses opposed: weak nuclear for Lemaire and strong nuclear for Gamow. Radioactivity, that is to say the spontaneous emission of radiation by matter, was discovered in 1896 by Henri Becquerel. As early as 1931, Lemaittre proposed his initially singular model of universe, the primitive atom, which, unstable, exists only for a moment, breaks up into pieces that break in turn and give rise to all the present components of the universe. He suggests that cosmic rays are the relics of the primitive universe. He advances a quantum origin of the universe, he introduces the idea of a cold big bang, that is to say an expansion resulting from the radioactive decay of an atom-universe. He first had a slowly evolving model related to the intensity of the weak force, responsible for the disintegration, then, based on the new knowledge of atomic physics, he turned towards a faster cosmology, with an explosive origin. He gives his cosmology a more structured version in 1933, published in French under the title "L'Univers en expansion" in the Annales de la Société scientifique de Bruxelles. In this text, Lemaitre demonstrates, among other things, the occurrence of singularities in the homogeneous relativistic cosmological models [2].

In return, Gamow proposes in 1946 the cosmological nucleosynthesis. In 1948, Alpher, Bethe and Gamow calculate the abundances of the elements formed in the primitive universe. The same year, Alpher and Herman predicted cosmic background radiation in the form of a black body at a temperature of 5 K. In 1952, Baade revises the extragalactic distance scale, which increases the cosmic time scale by a factor of 2.6. In 1965, Penzias and Wilson discovered the background radiation at a temperature of $3 \mathrm{~K}$. Dicke, Peebles, Roll and Wilkinson gave the cosmological interpretation of it in the framework of big bang models [2]. In essence, it can be said that the discovery of cosmic background radiation is due to the hot big bang model that Gamow and his team have achieved.

It's important to note that there is no direct succession between the work of Lemaître and that of Gamow. Nuclear physics was Gamow's inspiration here-not at first the influence of the prior mathematical work of Lemaitre or even Gamow's first teacher, Friedmann. Gamow had read Lemaître's, de Sitter's, 
and Eddington's papers on expanding models, but initially he did not apply them to his physics of the early universe. At the end of 1946, Gamow and Alpher decided to develop the rather abbreviated ideas that Gamow had issued during the year on primordial nucleosynthesis at the beginning of an expanding universe. Specifically, this meant working out hydrogen and helium could be developed out of the decaying neutron gas of the early phase of the universe. This led to Alpher and Gamow's 1948 paper, also known as the $\alpha \beta \gamma$ paper, a paper that has become a milestone in big bang history—and one that, like Lemaitre's and Friedmann's important papers, was almost entirely overlooked at the time. It was only in 1948, with the $\alpha \beta \gamma$ paper, that Gamow and Alpher and Herman realized that the model they wanted needed to originate in the Lemaitre model-and more importantly, it had to originate in a hot state, not in a cold nucleus as Lemaître had envisaged. Only a hot state of millions of degreed, they reasoned, could allow nucleosynthesis to "cook" elements like hydrogen, helium, and heavier elements [37].

Gamow, in other words, took Lemaitre's primeval atom and turned it into the big bang model that remains the base of the standard model to this day. One immediate consequence of such a hot big bang model, Gamow and his team realized, is that radiation from the primeval fireball should still remain, albeit at very attenuated wavelengths in the radio end of the electromagnetic spectrum. Where Lemaitre's atom model led him to examine cosmic rays as candidates for the leftover fireworks, Gamow's model more simply suggested a low hum of microwaves in the background of the universe.

\subsection{Theory of the Origin of Lemaître's Cosmic Rays}

In 1965, Penzias and Wilson discover the fossil radiation at the temperature of 3 $\mathrm{K}$, thus realizing the predictions of Gamow, Alpherer Herman on the primitive light. It has since been said that Lemaitre was not far from predicting the existence of cosmological black body radiation, photons emitted at a temperature of about $3000 \mathrm{~K}$ and cooled by a factor of 1000 by expansion, but that he will make the confusion with massive particles in cosmic rays [2]. It is now believed that most cosmic rays come from a number of sources such as the Sun, some stars, supernovae and their remains, neutron stars, and black holes. Does this mean that Lemaitre's suggestion that cosmic rays (massive particles) are relics of the early universe is false? We think, like Lemaitre, that cosmic radiation could have been created at the beginning of the world because these rays are endowed with energy of several billion electrons-volts and that we do not know any phenomenon currently taking place that is capable of such effects. What these rays might look like the most, are the rays that would be produced during decays of super-radioactive origin. They appear as the memory of the great initial transformations of the universe.

Lemaitre's thought on the origin of cosmic rays can be schematized by the following lines. The total energy of cosmic rays can be estimated at $10^{-34}$ grams 
per $\mathrm{cm}^{3}$. This value is based on the evaluation of the energy of the cosmic radiation falling on the Earth per square centimeter of surface and the transformation of this energy in mass by the relation $E=m c^{2}$. This energetic value, fairly secure in its order of magnitude, is a "density of equivalent mass". It should be compared with the density of matter, that is to say, what would be obtained if all the nebulae were vaporized and this matter was distributed uniformly in space. An order of magnitude of $10^{-30}$ grams per $\mathrm{cm}^{3}$ is found. Cosmic rays, assuming them uniformly distributed in space, have a considerable intensity since they are of the order of ten thousandth of all existing energy. It seems impossible to explain such energy, which represents one in ten thousand of all existing energy, if these rays have not been produced by a process that has brought into play all existing matter [38].

Imagine then ideal spheres that would be the neutral zones of attraction between neighboring nebulae. These spheres are expanding, since the whole system of the universe is expanding, the nebulae in each sphere are separating more and more. Cosmic radiation crosses these spheres, but, as the whole is symmetrical, we can imagine it bouncing on the surface of these spheres. With this image, we understand that the cosmic radiation must lose energy since it bounces on a body that flees. The calculation shows that this energy varies inversely with the radius of the sphere. So, assuming that cosmic rays occurred when the radius of the sphere was one-tenth of what it is now, then the energy of cosmic radiation would have reached not the ten thousandth, but the thousandth of the energy total of the universe. What assumes that cosmic rays come from far away and are not due to a nova or a small number of nearby stars. No satisfactory explanation is given for cosmic rays, which are 100 billion times more energetic than the others, and strike every $\mathrm{km}^{2}$ of the earth's surface about once every century. Models have already invoked ultra-energetic cosmic rays stemming from big bang, saying that Lemaître would not totally have been wrong on this question. Our model also plans ultra-energetic cosmic rays from the big bang. We believe that the hypothesis of fossil cosmic rays is just as true as the existence of cosmic black body radiation.

\subsection{Is Lemaitre's Cold Model as True as Gamow's Hot Model?}

We are then confronted with this other question: if we argue that Lemaitre's idea of fossil cosmic rays is just as truthful as the cosmic background radiation, does that mean that Lemaitre's universe model of a single cold quantum is just as true as Gamow's model of hot radiative universe?

At this point, it must be said that the first big bang models took into account only one force of nature, gravitation, described using the formalism of general relativity. Gravitation, attractive and of infinite scope, dominates on a large scale but is incapable of describing the physical conditions of the small-scale matter that prevailed at the beginning of the universe. General relativity constitutes a specific theory of gravitation, consequently incomplete. His equations lose all 
validity when the particles present in the primordial universe, endowed with gigantic energies, undergo other interactions than gravitation [33].

Lemaitre opposed, the first one, a "quantum cosmology" to this "relativistic cosmology". In 1934, he made the link between the cosmological constant and the vacuum energy of which he was the first to calculate the energy and to associate a negative pressure. However, he had no equation to explain the hypothesis of the single quantum. As for Gamow, he thought that some chemical elements had been produced during the first few minutes of the big bang and that the remaining radiation should be omnipresent. As a consequence of cosmic expansion, this original radiation must have cooled to a temperature of $5^{\circ}$ above absolute zero [25]. Just like Lemaître, he didn't have any equation for its "Ylem", this great Compression in a state of complete disaggregation from which the components had to emerge. They noted, Lemaitre first, the close correlation between observed expansion phenomena and certain mathematical consequences of Einstein's theory of general relativity. They had to fall back on the Friedmann-Einstein equation, and thus on the inadequacies of general relativity.

At the time of Penzias and Wilson's discovery in 1965 of background fossil radiation, the hypothesis of the primeval atom became, under the media name of big bang, a physical theory in its own right. The irony of history is that the radical novelty introduced by Lemaitre and Gamow, which consists in linking the structure of the universe on a large scale to the intimate nature of atoms, swims more than ever in the midst of speculation. Misunderstanding will persist as long as quantum mechanics remains irreconcilable with general relativity. The first can account for the quantum fluctuations that presided over the birth of the universe. It is the Planck era where the characteristic times of the phenomena are $10^{-43} \mathrm{~s}$. In contrast, Edwin Hubble's observations on the escape of galaxies can only be explained in the context of general relativity. The duration to take into account is the age of the universe, that is to say 15 billion years, or $10^{17} \mathrm{~s}$. There is therefore a factor $10^{60}$ between the two scales! The real is a ditch of 60 orders of magnitude that no theory currently can decrypt except the theory of Relation.

\section{A fundamental Equation}

\subsection{An Equation That Says Where Does the Time Come from}

We have said that Lemaitre had made the connection between the cosmological constant and the vacuum energy. The theory of the Relation, for its part, argues that vacuum energy, cosmological constant, dark energy and cosmological space-time wave constitute a single entity. They have the same flavor, color and smell, so that one can certify that they merge through cosmological time [33].

The cosmological time of this space-time is the key to the theory of Relation.

The time $t_{o}$ of the term $t_{o} c$ of the equation comes from $M_{V P}^{2}$ which represents the energy-matter. It emerges from the kinetic energy in dilation of protons, that is to say of a quantum cosmology. It is a physical time consubstantial with 
the universe which is born and an indicator of an energy propagated at the speed of light. The term " $t_{0} c$ " refers to a radius from the center point of a sphere created by the large initial boom (in this case, the Planck length of the Planck sphere, but it may shrink towards the absolute zero point). The image of the big bang as a cosmic explosion ejecting the material contents of the universe like shrapnel from an exploding bomb is a useful and non-misleading representation as long as these splinters make space-time instead of being in it. The big bang would be an eruption of compressed space-time-matter, whose deployment, like a shock wave, would still transport matter and energy [39].

This radial move is an electromagnetic wave. We can say that the radius of space-time belongs to the family of electromagnetic waves: the wavelength is the radius $\left(\sim 10^{26} \mathrm{~m}\right)$ of the universe and the period $\left(\sim 10^{17} \mathrm{~s}\right)$ is his age. Just like Maxwell's electromagnetic theory of light, the space-time wave is an oscillation wave of the electric and magnetic fields that propagate in space. We can call it electrogravitational wave or electromagnetic wave of space-time. It carries energy and momentum. In fact, it is the electromagnetic standing wave [40], the "radiation" at $2.7 \mathrm{~K}$, or the vacuum energy.

The equation gives a negative cosmological constant which prints a deceleration of the expansion of the universe. Its positive pressure exerts an attractive force. Does this deceleration of the universe affect the course of time? Physics formally distinguishes the course of time from the arrow of time. The course of time indicates to the fact that time passes and that by passing it produces duration. It generates the succession of events and is causal, because time goes in one direction without backtracking. The arrow of time presupposes the existence of a well-established course of time within which certain phenomena are themselves temporally oriented, that is to say irreversible: once accomplished, it is impossible to cancel the effects they produced. In the context of expansion, it seems that the course of time and the arrow of time ultimately proceed from one and the same reality.

Can we claim, with this relation where the time stemming from the energy-matter $M_{V P}^{2}$ links the physics of the infinitely large to that of the infinitely small, to explain the emergence of the irreversibility observed at the macroscopic scale from physical laws that ignore it at the microscopic scale?

We know that according to the equations of current physics, all the phenomena taking place at the microscopic level are reversible, they can unfold in one direction as well as in the other. The dynamics of the phenomena do not depend on the orientation of the course of time. We could call past what we call future, and vice versa, without affecting the physical process in which they participate. On the other hand, at our scale, we observe only irreversible phenomena, arrowed phenomena. The oldest explanation is based on the second principle of thermodynamics, according to which any physical system evolves in general without returning to its initial configuration: lukewarm water never becomes hot water on one side, cold water of the other. However, it seems convincing that if 
the universe, after its expansion, entered the contraction phase, it would imply a reversal, not of the course of time, but of the arrow of time. The irreversibility of expansion would only be in fact, not in principle [41].

What is the true origin of cosmological time? Our equation, as well as the equations of traditional cosmology (derived from the Friedmann-Lemaître equation), allow us to go back to the Planck scale, where, below, the usual representations of space and time lose all meaning. Admittedly, the theory of the cyclic universe makes it possible to envisage a "pre-time" different from the usual (reversible) physical time, but this notion only moves a link in the chain of causality that goes back in time, a chain that either has no beginning, or ends in a first cause. We can call "zero time" this first moment which corresponds to a situation where the equations begin to be valid. This first moment is not quite one, in the sense that corresponds in no way to the "absolute zero instant" of the origin of the universe.

How did cosmological time begin? The expansion of the universe becomes the real engine of time. Our model of mathematized cosmos is unambiguous: the universe decelerates. This deceleration of the expansion goes against the phenomenon of acceleration endowed with a positive cosmological constant which gives rise to a universal repulsive force with a density of the energy of the vacuum which remains the same-what seems absurd to us-, while ordinary matter is diluted and ends up being unable to slow down [42]. It seems certain that this is not the case in the theory of Relation. What is revolutionary in the latter is that in the process of expansion, the energy of the vacuum at the beginning was to be $10^{120}$ times higher than today where the density of the cosmological constant is almost equal to zero. It dilutes and ends up slowing expansion. We have a negative cosmological constant which gives rise to a universal attractive force [6]. On the other hand, ordinary matter (which is the potential energy of our equation) had to have a gravitational mass almost zero at the beginning, it strengthens with expansion and its attractive effect always increases.

Neither general relativity, nor quantum physics, nor a possible synthesis of the two, can today describe the apparition of the universe as a physical event. How could they do it when they consider that the passage of time is an illusion? The reality defined by the special relativity is a four-dimensional space-time block where it is impossible to define a "now". General relativity says that this block is actually a space-time-force-matter block where the force-matter content is nested in the space-time container that it distorts by its presence. Quantum theory says this block is multiple.

The notions of universal time and the oneness of the reality do not exist for these theories while they are at the heart of the theory of Relation. The conceptual leap was to introduce the notion of temporal flow and that of temporal orientation (or temporal arrow). This temporal arrow implies privileged strata that correspond to the idea of a "stratum of the now" that would move towards the future as if a projector successively illuminated the "strata of density" of 
space-time [43]. The course of time, as well as the arrow of time, would result from the fundamental cosmic temporal irreversibility of expansion and would correspond to emerging properties of quantum cosmology [44].

The universe erupted from a singular energetic event, which gave birth to all space and all matter. Our equation goes back to that "zero instant" from which cosmological time emerges. This zero instant corresponds to the $10^{-43} \mathrm{~s}$ which followed the big bang, known as Planck time where the temperature is estimated at $10^{32}$ Kelvin degrees. It also coincides with the maximum increase in quantum mass obtained by relativization, or the transformation of Lorentz, from $v^{2}$ to $c^{2}$. If the expansion decelerates, that is to say if the engine of time decreases, its speed, the speed goes from $c^{2}$ to $v^{2}$, and the course of time should itself "decelerate".

Energy spreads by creating space-time, and cools. We can see this freeze in the formula in two ways, because there are two speeds. First, a drop in the speed of " $M_{V P}^{2}$ " caused a gradual drop in temperature and a slowing of the rate of expansion, and a concomitant increase in gravity. The fermions, a priori in the state of radiation, maintained their maximum speed until about 300,000 years and the decoupling of the matter from the radiation took place when the speed passed under $c$. The arrow of time, which refers to the possibility that things have of knowing over time of irreversible changes or transformations, is in our equation a property of material phenomena since it originates from $M_{V P}^{2}$. Secondly, the velocity $c$ of the electromagnetic wavelength of space-time: when the universe became that of today, the kinetic energy and the density of the radiation diminished. The space-time line " $t_{o} c$ " conveys a field of less and less energetic bosons that propagates at the speed of light. Each second contains less energy-event, and the universe-as a whole-no longer changes significantly over the seconds.

\subsection{Planck Units; Wall of Planck}

At Planck time, that is during the oldest period of the universe which our only equations manage to conceive, the universe was nervous and dry, tiny and full of energy, and its space-time had a weird structure. The Planck wall is expressed in the form of a time, a length and a characteristic energy. Planck's wall refers to circumstances in which the quantum and gravitational phenomena begin to really overlap. His description must involve together the constant of the gravitation $G$, the speed of the light $c$ and the constant of Planck $h$. Their combination leads to the following results:

Planck energy is given by the expression $\left(h c^{5} / G\right)^{1 / 2}$. It is worth ten billion billion times the mass energy of a proton, or $10^{19}$. Matter at that time was furiously agitated.

Planck length is given by the expression $\left(h G / c^{3}\right)^{1 / 2}$. It is about $10^{-35}$ meters, which is seventeen orders of magnitude less than the size of a quark or an electron. It is interpreted as saying that below this scale of distance, the notion of 
space as we describe it in our physical theories no longer makes sense. Planck time is given by the expression $\left(h G / c^{5}\right)^{1 / 2}$. It is worth about $10^{-43}$ s. Planck wall applies to the universe as it was $10^{-43} \mathrm{~s}$ after the big bang [33]. The time $t_{o}$ used at the Planck scale is a time during which the nascent universe was governed by quantum effects, a time during which the quantum and relativistic gravitational effects of Planck's units simultaneously become comparable. This suggests that the correct description of space-time-matter in the primordial universe requires a theory combining relativistic gravitation and quantum mechanics [45].

The theory of Relation offers an equation that integrates the two theories. We wrote it in (2) above [ $k e^{2}=M_{V P}^{2} t_{o} c$, where $M_{o p}$ is rest-mass; $M_{o p}\left(1 /\left(1-v^{2} / c^{2}\right)^{1 / 2}\right)$, or $M_{v p}$, is rest-mass + kinetic energy $\left.(T)\right]$. Particles come in pairs, each with a counterpart antiparticle:

$$
\pm k e^{2}= \pm\left[M_{o p} /\left(1-v^{2} / c^{2}\right)^{1 / 2}\right]^{2} t_{o} c .
$$

In our time $(v=2 / 3 c)$, it becomes

$$
2.3 \times 10^{-28} \mathrm{~kg} \cdot \mathrm{m}^{3} \cdot \mathrm{s}^{-2}=\left(2.2439 \times 10^{-27} \mathrm{~kg}\right)^{2}\left(1.528 \times 10^{17} \mathrm{~s}\right)\left(3 \times 10^{8}\right) .
$$

But at the time of Planck's values, the antagonism between the two theories is appeased and they are brought together for the first time. If we apply

$$
\pm k e^{2}= \pm M_{V P}^{2} t_{o} c= \pm M_{V P}^{2} h / m_{o} c= \pm M_{V P}^{2} 2 G M^{o} / c^{2},
$$

the mass of the "baryon-proton" $M_{v p}$ will be

$$
1.479 \times 10^{3} \mathrm{~kg}\left(2.3069 \times 10^{-28}=M_{V P}^{2} 3.51 \times 10^{-43} c\right) .
$$

The wavelength

$$
\lambda=t_{o} c=R=h /\left(2 \pi m_{o} c\right)=\hbar /\left(m_{o} c\right)=1.05458 \times 10^{-34} \mathrm{~m} .
$$

We use $\hbar$ with the Planck time $(\hbar / c)$ and the Planck length: this is consistent with $t_{o} c$, which is linear, not circular. [It may seem odd to say that wavelength $1.05458 \times 10^{-34} \mathrm{~m}$ numerically equals the value of $\hbar$ in J sec $\left(1.05458 \times 10^{-34}\right)$. We explain it from the relation $E t=h ; E t=2 \pi \hbar ;\left(m_{o} c / 2 \pi\right) t_{o} c=\hbar$. Planck's mass $\left[k e^{2}=M_{V P}^{2} h /\left(m_{o} c\right) ; 2.3069 \times 10^{-28}=2.1874 \times 10^{6} h /\left(m_{o} c\right) ; \quad m_{o}=2.0958 \times 10^{-8}\right]$, in the expression $\left(m_{o} c / 2 \pi\right)$ gives 1 , hence $t_{o} c=\hbar$.]

With the de Broglie wave that travels at the speed of light as that of the particle $m_{o}$, the boson $m_{o}$ gives $2.09 \times 10^{-8} \mathrm{~kg}\left(k^{2}=M_{V P}^{2} h / m_{o} c\right)$. We employ $h / m_{o} c$ because quantum mechanics describes a particle, not a radius. Everything happens as if the light consisted of grains, and each grain of light had an energy proportional to the frequency $v$ of the light: $E=h v$. We write $\varepsilon=\hbar \omega=2 \pi \hbar v=h v \quad(\omega$ is the pulsation while $v$ is the frequency; $\omega=2 \pi v)$. We have just equated $\hbar$ with a wavelength, a distance, a radius, it goes without saying that $2 \pi \hbar=h$ represents the circumference of a "quantum" of radiation energy, which constitutes a particle, hence the use of $h / m_{o} c$.

With $k e^{2}=M_{V P}^{2} 2 \pi G M^{o} / c^{2}$, general relativity determines the mass of the universe at Planck time, $M^{o}=2.26 \times 10^{-8} \mathrm{~kg}$. We utilize $2 \pi G M^{o} / c^{2}$ (not 
$G M^{\circ} / c^{2}$ ), considering that the term describes a mass with a circumference, not a radius.

Instead of having $M_{\text {Planck }}=(h c / 2 \pi G)^{1 / 2}=2.1768 \times 10^{-8} \mathrm{~kg}$, which seems to be one of two similar masses, we have $M^{o} m_{o}=h c / 2 \pi G$, which are two different masses: $m_{o}=2.09 \times 10^{-8} \mathrm{~kg}$ of quantum theory and $M^{o}=2.26 \times 10^{-8} \mathrm{~kg}$ of general relativity. The Planck mass $2.1768 \times 10^{-8} \mathrm{~kg}$ is actually the average of these two distinct masses $\left(M^{o} m_{o}\right)^{1 / 2}$. Their numerical value corresponds to Planck mass $\left[(\hbar c / G)^{1 / 2}=2.17682 \times 10^{-8} \mathrm{~kg}\right]$ and they are reminiscent of the famous hidden variables.

\subsection{The New Variable: $M_{V P}^{2}$}

The new parameter $M_{V P}^{2}$, or $\left[M_{o p} /\left(1-v^{2} / c^{2}\right)^{1 / 2}\right]^{2}$, is an essential element. Its value changes throughout the expansion. The Lorentz transformation of this variable [12] inscribes the equation in a relativistic cosmology (although our mathematical model is central and global whereas general relativity is above all peripheral and local). The velocity $v$ of this transformation, starting from the speed of light and moving towards 0 (it would be about $2 / 3 c$ today), constitutes a variable velocity of light. Thus the limit of a signal it was thought up till now to be that first measured with the light waves was much greater at the beginning of the history of the universe.

The value of $M_{V P}^{2}$ (two protons relativized with a speed close to light) at Planck time is confused with the "isotope of the neutron" (also called "unique quantum") of Lemaitre or with the primitive atom of Gamow. A gigantic kinetic energy is stored in this very dense and very hot atom in the form of an electromagnetic mass. When all this energy is released, it appears in the universe in two forms: on the one hand the energy of the electromagnetic radiations, without mass; on the other hand, energy brought into play by mass movements, kinetic energy.

In our equation, $t_{0} c$ represents the emission of electromagnetic radiation, without mass. During the 380,000 years that followed the big bang, light could not propagate freely in space: the density of matter was such that photons never ceased to interact with particles of matter, so that the universe was an opaque medium to its own light. Its continuous cooling, however, eventually caused a phase change after 380,000 years of expansion, when the temperature of the universe was only 3000 Kelvins: the electrons were captured by nuclei, forming electrically neutral atoms. Since photons interact little with atoms, they can propagate freely in the universe, without encountering obstacles at every step. This radiation, which has been liberated from matter, now constitutes what is called the "cosmic microwave background" (detected in 1964 by Arno Penzias and Robert Wilson). It is the light predicted by the hot atom of George Gamow. It is the light predicts by George Gamow's hot atom [33].

In the equation $k e^{2}=M_{V P}^{2} 2 \pi G M^{\circ} / c^{2}$ at Planck time, $M_{V P}^{2}$ represents the 
kinetic energy of the universe while $M^{o}$ of $2 \pi G M^{\circ} / c^{2}$ represents its potential energy. During the initial explosion, the first stage of the big bang, $M_{V P}^{2}$ releases the extraordinary mass of inertia (due to resistance to movement, resistance to acceleration) that it contained. In the minutes following the big bang, some nuclear reactions occurred. Helium was then synthesized. The first stages of the expansion consisted of a rapid expansion determined by the energy-matter of the entire universe condensed in the initial quantum of Lemaitre, roughly equal to the mass of the universe. If we admit that cosmic radiation was emitted during the first fragmentations of the universe, it would correspond to transformations of the kind that accompany the radioactive phenomena that we know, but with a considerably greater generality. Often, during the same phenomenon, radioactivity manifests itself in the form of disintegration, $x$ endowed with mass, on the other hand in the form of electromagnetic energy, the emission of a ray $\gamma$, without mass. The ratio between the wavelength of space-time and the wavelength of cosmic gamma rays is $\left(10^{26} \mathrm{~m} / 10^{-14} \mathrm{~m}\right) \approx 10^{40}$.

Thus the cosmic rays would be the witnesses of the primitive activity of the cosmos, and it would have preserved, for billions of years, in the empty space, the memory of the super-radioactive age. We endorse Lemaitre's hypothesis, and it is probable that it will eventually be verified.

\section{Discussion on the Model of the Theory of Relation and That of the Classic Big Bang Theory}

All the models proposed by Lemaitre, and consolidated by Gamow, concerning the expansion of space refer to the Einstein-Friedmann equation. The relativistic cosmology that emerges is at the origin of the standard cosmology of the big bang which succeeds in giving the approximate age of the expanding universe, its previous and future history, as well as, in our view, the irrational drift of the acceleration of the expansion. However, the first big bang models only take into account gravitation, described using the formalism of general relativity. They have nothing to do with quanta and electromagnetic interactions, strong nuclear and weak, which determine the behavior of the matter at the beginning [33].

The equation that we propose, developed in a previous work [3], although it has nothing to do with the equation of Einstein-Friedmann, gives results of the same order on the age of the universe, as well as with the results obtained by Hubble or by the analysis of the cosmic microwave background. It is truly suitable for "quantum cosmology": it has its source in quantum mechanics and also relies on relativistic cosmology derived from the theory of general relativity. It brings a different theoretical background on the quantum origin of the universe, integrates an irreversible cosmological time, gives a new light on the cosmological constant, the energy of the vacuum and the dark energy. It has the advantage of being able to teach us a lot about the internal structure of the universe, since this structure is included in the model. It reveals the fundamental role played by the energy of the quantum vacuum, both in the birth process of the universe and 
in the expansion phase resulting from it. It introduces negative energy. It predicts an expanding universe in constant deceleration and contradicts the accelerated expansion phase that currently appears to be in effect.

The quantum cosmology of the theory of Relation aims to describe the evolution of the universe as a whole from a quantum point of view through a "space-time wave function of the universe", but does not necessarily imply the quantification of gravitation. It must be recognized that quantum cosmology is not a theory of quantum gravity. The latter seeks to describe phenomena on a very small scale and does not necessarily imply the description of the universe as a whole. Several hypotheses are candidates for quantum gravity, although we aspire to a unique, coherent and conceptually satisfactory theory, of which quantum mechanics and general relativity are only valid approximations in their respective domains [45].

In an article in the journal Astrophysical Journal [46], a team of astronomers led by Fred Hoyle asserted that it is possible to design a cosmology without resorting to the classical big bang theory. Already in the fifties, Fred Hoyle had supported a theory opposed to that of the big bang, known as the theory of "stationary state" or "continuous creation". He came back, more than forty years later, with a model partly identical to the one he had to abandon. With a difference in size however: if the idea of continuous creation is saved, it no longer occurs anywhere in space but in privileged places. And there would not have been an initial explosion but infinity of mini-big-bang. And here is transgressed the taboo theory of the big bang by astronomers who claim that there was not a unique and creative big bang but repetitive mini big bang that make the history of the universe an eternal re-beginning [47].

In a very broad way, our model considers the universe as a fragment of multiverse, which is like a soup pot boiling perpetually; continually forming new bubbles. These eventually grow and burst, but the soup jar is eternal. Each of them is a universe started by a big bang. Often these bubbles-big bang come from internal bubbles of the pot which ended in big crush. The latter becomes the big bang of a universe like ours. Thus can be explained the contradictory duality of our universe. Our universe was given birth by another universe which continues its childbirth by disintegrating in our universe.

Everything happens as the two universes coexisted, as if there were two universes in one, ours which is formed on the account of a universe that never stops disintegrating [5]. Because of the inversion of the arrow of cosmological time, based on the laws of the thermodynamics, the energy of the world which contracted is negative in relation to the positive energy of our expanding world. Yet, on the grounds that the existence of particles of negative energy would not be in accordance with the observable reality, it is half of our universe that is denied [48].

The existence of particles of negative energy has been scratched by a stroke of pen by most physicists. It is nevertheless part of the results obtained by Dirac in 
his formulation of the equations of quantum mechanics [49]. These equations, which remain the foundation of quantum theory, reveal a continuum of states of negative energy. If we consider the binding energy of a hydrogen atom, the equations describe a whole series of possible energies, with a minimal state of energy. Below this state of minimum positive energy, the energy becomes zero over a very long vacuum interval, then reappears in negative form, and develops in the other direction with infinity of states. From a conventional point of view, these states of negative energy are considered supernumerary and superfluous, but for Dirac, these states had a reality. It is by calculating from these states of negative energy that he predicts the existence of the positron. The experiment gave him reason. This arbitrary decision by physicists to discard this type of particle of negative energy, explicitly provided by the Dirac equations, today distorts all the reasoning of cosmological physics. This question was the subject of two previous articles [32] [50].

Astrophysicists would do well to question the basis of their creed. Besides starting from a beginning of which we know nothing, whether by observation or experimentation, that it does not meet the basic criteria of science, the standard big bang theory is contradicted by several current observations, which are ignored. For example, the subtle observations made by astronomers at the Burakan Observatory (of the Armenian Academy of Sciences), of which Viktor Ambartsumian was the director, have been left aside. The work of Armenian astronomers confirmed the new hypothesis of star formation according to which the evolution was from condensation to rarefaction, from hyperdense bodies to less dense bodies. They further confirmed that the birthplaces of the new galaxies were the centers of the old galaxies. The latter swell, from time to time, show a tendency to divide and eject powerful gas clouds containing free electrons that explain the powerful radio-ray emission. Rapid protons give birth to cosmic particles. They confirmed the theory of the division of galaxies: they noticed that in some cases, from the center of a giant galaxy escaped a jet that ended in a dwarf galaxy whose color, unlike the "old galaxies", yellow and red, was blue. It was a birth of a small galaxy by a big one. Over time, the jet binding - a kind of "umbilical cord"-should disappear, giving the "offspring" the opportunity to start an independent life. Such dwarf galaxies were discovered near many supergalaxies [51] [52].

Because standard theory cannot explain certain phenomena, its advocates have developed an ability to systematically ignore these facts [53]. Observations show, contrary to the Lemaitre-Gamow postulate, that stars and galaxies have not structured in one go and that their age can be very different. Does this mean that we must, however, invalidate the hypothesis of the primitive atom for the entire cosmos? Certainly not.

We believe that our universe undergoes both processes. This would be explained as follows: About 15 billion years ago (at the Planck scale, and not at absolute zero), the primary matter, then dense and hot, began its expansion. The 
expansion would have been preceded by a period of contraction and it would not have occurred at the same time for all the matter. In the world in contraction, some of the matter has contracted faster than the other, giving the big crush which is for us the big bang. The first process would be that the kinetic energy of the primordial explosion that spawned the expansion would be counterbalanced by the gravitational attraction that caused in the early days of the universe the formation of much of the condensed objects (atoms, molecules, stars...). The sequence density variation-gravitational collapse-accretion would be the prerogative of the standard model. The second process concerns isolated pieces of material from the pre-universe that contracted and that would have been delayed in their appearance and development. These would be pre-existing hyperdense material nuclei as a starting point for galactic or stellar formations. These phenomena are observed and localized in time and space. They would come from the latent matter which, at the end of a certain time, appears, expands in turn, begins to interact with the surrounding environment, generating the hotspots of intense radiation and violent patterns that we are currently seeing. The violent explosive effect of a strong negative pressure within a localized region where an important mechanism of creation has occurred, for example active galactic nuclei, produces an ejection from this region.

Photos taken by specialists have shown the phenomenon of ejection. Examples have been provided. There are a large number of objects whose figures give examples of direct evidence that discrete objects-galaxies or quasars-with a strong redshift are ejected from low redshift galactic nuclei. Standard theory offers no explanation for this type of phenomenon, which its defenders have continued to ignore. Yet these are real facts.

Even if we accept with caution the hypothesis of Ambartzoumian, according to which these stellar formations would have for origin a prior concentration of hyperdense matter (nuclear plasma) in proto-stars, it is nevertheless true that, often, everything takes place, not as if stars clustered into galaxies, but rather as if the stars were formed somehow from a galactic core (itself hyperdense) since the galaxies, composed of billions of stars, then develop in the aspect that we know them, with their own rotational movement. Physicist Milne has already tried to reconcile the thesis of the primitive atom with the continuous formation of stars or galaxies and with their evolution. While retaining the idea of an initial explosion of all matter concentrated "somewhere" at the instant "zero"-explosion causing by molecular dispersion the creation of a space in constant expansion over time, Milne admitted, however, that the particles were grouped progressively to form stars then stellar systems. Subsequent observations have shown that stars are born in groups within galaxies, and then separate from each other.

The above development would seem to imply-if we accept Ambartzumian's conception of the prior existence of proto-stars-that hyperdense nuclei have themselves preceded the formation of galaxies. The proto-stars could be only fragments of these nuclei, which, by splitting themselves primitively, could have 
given rise to the formation in groups (or at least in pairs) of galaxies. This, which is no longer a simple view of the mind, concurs, in a certain way, with Lemaitre's cosmogonic hypothesis in 1931 according to which the present world has resulted from the radioactive decay of an atom. Guided by thermodynamic considerations that sought to interpret the law of the degradation of energy within the framework of quantum theories, the discovery of the universality of radioactivity, since then, makes more plausible its suggestion which assigned a radioactive origin to all the existing cosmic matter as well as the most powerful cosmic rays that would be the relics of the primitive universe.

\section{Conclusions}

The year 1998 is that of a spectacular twist: the experimental discovery of the acceleration of the expansion of the universe. Astrophysicists have observed several distant supernovae of type 1a acting as luminous stallions. The results indicate that they seem more distant than expected. Their position suggests that the expansion of the universe would have been accelerating for at least six billion years. On the other hand, the very distant galaxies in which the type 1a supernovae explode show a slowing of their recession velocity, sign that the expansion of the universe has been slowed down in the first billions of years. They concluded overnight without discussion that the acceleration of distant supernovae would result from a dark, hypothetical and invisible energy, which could be a quantum vacuum energy in the form of a positive cosmological constant. The problem is that the value of the quantum vacuum energy deduced from astronomy, which seems to be $10^{120}$ times too high compared to what the observations indicate and to the value calculated by the theoretical physicists. Explicitly and approximately, the vacuum energy density proposed by quantum field theory is about $10^{121} \mathrm{GeV} / \mathrm{m}^{3}$, which corresponds to about $10^{121}$ protons $/ \mathrm{m}^{3}$. The value of the current cosmological medium is $10 \mathrm{GeV} / \mathrm{m}^{3}$ or about 10 protons $/ \mathrm{m}^{3}$. The gigantic gap between the two is what is called the vacuum catastrophe [31] [33].

In our view, we are witnessing a farce of official science. First, the technique of measuring astrophysical distances using supernovae cannot be trusted. Astronomers assume that the intrinsic brightness of the supernovae is the same for all, independent of the particular object being measured. This hypothesis, impossible to prove, is free. The chemical composition of the first supernovae was necessary of a composition different from that of now since the generations of stars had not yet succeeded one another to make the heavy elements. Then, considering what physicists know about radioactivity and cosmic radiation, the researchers thought they were right that the cosmological constant should be several orders of magnitude larger than the density of ordinary matter. It was enough to introduce into the equations a simple parameter, dark antigravitational energy appeared and it is done. The fact is that cosmological observations indicate a low vacuum energy and a cosmological constant with a near zero density [21].

The problem of the cosmological constant (or the vacuum energy) constitutes 
the greatest challenge of contemporary theoretical physics. Many theoreticians sense that the resolution of this major conflict could perhaps lead to the unification of gravity and quantum theory. For more than half a century, two tracks have been followed to quantify gravitation. The string theory which favors the geometric approach of general relativity and the theory of loops which prioritizes the quantum approach of fields. Whatever may be said about these two promising theories, and other approaches, these have not yet yielded results in terms of prediction or experimentation. Moreover, they lack a basic, coherent and unique equation, which translates simple laws leading to unification.

We claim to have such an equation whose master key is time. If the mathematical story told by the standard model springs from a mathematical singularity, the equation presents a non-singular physical beginning in the form of a "fireball" which represents the physical birth certificate of the universe. This material content that cools when expanding gives birth to space-time. The time $t_{o}$ of the expression $t_{0} c$ makes impasse on the question of the genesis (initial singularity) to concern itself only with the immediately subsequent cosmological events (Planck era). The equation of theory of Relation therefore presents a universe governed by the laws of mathematical physics that incorporates a time that becomes a unit of measurement, which gives a thermal history and legitimacy to the existence of things. It bridges the gap of $10^{60}$ orders of magnitude separating the quantum mechanics of the subatomic world from the astronomical scale of general relativity by a cosmological time that extends between a time of $10^{-43} \mathrm{~s}$ and a time of $10^{17} \mathrm{~s}$. It is proof that both quantum theory and general relativity are wrong about the nature of time that constitutes cosmological history [31] [54].

This cosmological time which defines the notions of space and time between this beginning of space-time and now gives meaning to the laws of physics as we know it. It merges with the vacuum energy, or a dark energy, that empties the universe by never ceasing to dilute itself in favor of ordinary matter. The model of the theory of Relation also attempts to reconcile the dynamic aspects of the big bang theory with the eternal nature of continuous creation. It strives to create a bridge between the competing cosmological models: the idealistic models that presuppose the creation of an incredible density nucleus containing all the matter and the energy of the universe, which nucleus would have disintegrated once for all, and the materialistic models that conceive pre-existing hyperdense nuclei as a starting point for galactic or stellar formations.

We believe that this equation, which contrasts with the current popular view of cosmology and cosmogony, is closest to the equation that Lemaitre lacked to defend his "primeval atom hypothesis" and his prediction of fossil cosmic rays, witnesses of the primitive activity of the cosmos.

\section{Conflicts of Interest}

The author declares no conflicts of interest regarding the publication of this paper. 


\section{References}

[1] Lemaitre, G. (1931) Monthly Notices of the Royal Astronomical Society, 91, 490-501. https://doi.org/10.1093/mnras/91.5.490

[2] Luminet, J.-P. (2004) L'invention du Big Bang. Éditions du Seuil, Paris, 111-126, $151,159,163,194,196,203,210$.

[3] Bagdoo, R. (2019) Journal of Modern Physics, 10, 310-343. https://doi.org/10.4236/jmp.2019.103022

[4] Stein, J.D. (2011) Cosmic Numbers. Basic Books, New York, 199-201.

[5] Bagdoo, R. (2008) The Pioneer Effect: A New Theory with a New Principle. http://vixra.org/abs/0812.0005 https://www.academia.edu/5535864

[6] Bagdoo, R. (2011) Cosmological Inconstant, Supernovæ 1a and Decelerating Expansion. https://www.academia.edu/5539777 http://vixra.org/abs/1304.0169

[7] Guy, J., et al. (2010) Astronomy \& Astrophysics, 523, A7. https://doi.org/10.1051/0004-6361/201014468

[8] Blanchard, A. (2005) Cosmological Interpretation from High Redshift Clusters Observed within the XMM-Newton $\Omega$-Project. Proceedings of the International Conference DARK 2004, College Station, 3-9 October 2004, 34-46.

[9] Vishwakarma, R.G. (2003) Monthly Notices of the Royal Astronomical Society, 345, 545-551. https://doi.org/10.1046/j.1365-8711.2003.06960.x

[10] Vishwakarma, R.G. and Narlikar, J.V. (2010) Research in Astronomy and Astrophysics, 10, 1195-1198. https://doi.org/10.1088/1674-4527/10/12/001

[11] Kowalski, M., et al. (2008) The Astrophysical Journal, 686, 749-778.

[12] Nielsen, J.T., Guffanti, A. and Sarkar, S. (2016) Scientific Reports, 6, Article No. 35596. https://doi.org/10.1038/srep35596

[13] Crew, B. (2016) No, The Universe Is Not Expanding at an Accelerated Rate, Say Physicists, 24 Oct. https://www.sciencealert.com

[14] European Space Agency News Release (2003) Has XMM-Newton Cast Doubt over Dark Energy?

[15] Hubble, E. and Tolman, R.C. (1935) Astrophysical Journal, 82, 302. https://doi.org/10.1086/143682

[16] Kaku, M. and Trainer, J. (1987) Beyond Einstein. Bantam New Age, New York, 10-20-1, 30-1, 35.

[17] Will, C.M. (1986) Was Einstein Right? Basic Books Inc., New York, 153, 166-167.

[18] Gamow, G. (1963) La Gravitation. Payot, Paris, 134-139.

[19] Bramand, P., Faye, P. and Thomassier, G. (1980) Physique, Terminale C, E. Eurin-Hachette, Paris, 52-55.

[20] Nottale, L. (1997) L'Univers et la lumière, Champs. Flammarion, Paris, 141.

[21] Magnan, C. (2011) Le théorème du jardin. amds édition, 127, 160-1, 168-9, 247-8, 263.

[22] Narlikar, J.V. (1986) Une gravitation sans gravité. Payot, Paris, 175.

[23] Orear, J. (1967) Fundamental Physics. John Wiley \& Sons, Inc., New York, 87, 100, 156, 284-287.

[24] Davies, P.C.W. and Brown, J. (1988) Superspring. Cambridge University Press, 
Cambridge, 5, 26, 27, 47.

[25] Silk, J. (1997) Le Big Bang. Éditions Odile Jacob, Paris, 10.

[26] Moffat, J.W. (2009) Reinventing Gravity. Thomas Allens Publishers, Toronto, 121, $122,162,206-208$.

[27] Michaud, A. (2017) Electromagnetic Mechanics of Elementary Particles. 2nd Edition, Scholars' Press, Berlin, 205-223.

[28] Hawking, S.W. (1988) Physics Today, 41, 115. https://doi.org/10.1063/1.2811637

[29] Klein, É. (2018) Les secrets de la matière. Édition j'ai lu, Paris', 83-84.

[30] Narlikar, et al. (2002) PASP, 114, 1092. https://doi.org/10.1086/342374

[31] Gunzig, E. (2008) Que faisiez-vous avant le big-bang? Odile Jacob, Paris, 162, 200, $218,218,219$.

[32] Bagdoo, R. (2019) Journal of Modern Physics, 10, 163-175. https://doi.org/10.4236/jmp.2019.102013

[33] Klein, É. (2010) Discours sur l'origine de l'univers. Champs Sciences, Paris, 34-36, $46,57-59,65,66,116-118$.

[34] Landa, P. (2016) Georges Lemaitre: The Big Bang Theory and the Origins of Our Universe. 50Minutes.com, USA Columbia, SC, 16-18.

[35] Lemaître, G. (1945) L'hypothèse de l'atome primitif, Actes de la Société Helvétique des sciences naturelles. 77-96.

[36] Gamow, G. (1961) La création de l'univers. Dunod, Paris, 30-36.

[37] Farrell, J. (2010) The Day without Yesterday. Basic Books, New York, 135, 136.

[38] Leprince-Ringuet, L. (1948) Les rayons cosmiques. Albin Michel, Paris, 349, 350.

[39] Greene, B. (1999) The Elegant Universe. Vintage Books, Random House, Inc., New York, 83.

[40] Pagels, H.R. (1982) The Cosmic Code. Bantam New Age Book, New York, 5, 9, 237-243.

[41] Klein, É. (2009) Le facteur temps ne sonne jamais deux fois. Flammarion Champs Sciences, Paris, 41, 164, 165. https://doi.org/10.14375/NP.9782081220225

[42] Susskind, L. (2007) Le paysage cosmique, folio essays. 235, 251, 252.

[43] Damour, T. (2016) Si Einstein m’était conté... Flammarion, Champs, Paris, 153, $252,277,278$.

[44] Klein, É. (2009) Les tactiques de Chronos. Flammarion, Champs essais, Paris, 43, $126,128,153,177,178$

[45] Luminet, J.-P. (2006) Le Destin de l’Univers ll. Librairie Arthème Fayard, Paris, 561, 563-568.

[46] Hoyle, F., Burbidge, G. and Narlikar, J.V. (1993) The Astrophysical Journal, 410, 437-457. https://doi.org/10.1086/172761

[47] Andrillat, H. (1994) Science \& Vie, No. 916, 33-35.

[48] Petit, J.-P. (1997) On a perdu la moitié de l'univers. Edition Albin Michel, S.A., Paris, 10,11 .

[49] Davies, P. (1988) The Forces of Nature. Cambridge University Press, Cambridge, 53-58.

[50] Bagdoo, R. (2013) The Energy in Virtue of the Principle of Compensation. http://vixra.org/abs/1301.0180

https://www.academia.edu/5539802 
[51] Hoyle, F., Burbidge, G. and Narlikar, J.V. (1998) Le big bang, une conception bien fumeuse. In: La Recherche, Hors Série Cosmologie No. 1, 104-108.

[52] Radounskaïa, I. (1972) Les idées “folles”. Editions Mir, U.R.S.S., Moscow, 145, 366-374.

[53] Cuny, H. (1961) Astronomie d'aujourd'hui. Editions La Farandole, Paris, 62-68.

[54] Smolin, L. (2006) The Trouble with Physics. Mariner Books, Wilmington, 256. 